\title{
Incidencia de las TIC en la Comercialización de los Productos de las Comunidades Rurales Indígenas en el Ecuador
}

Lupien, P., M. Posso, A. Basantes \& G. Chiriboga. 2020. Incidencia de las TIC en la Comercialización de los Productos de las Comunidades Rurales Indígenas en el Ecuador, Canadian Journal of Latin American and Caribbean Studies. 45(1).

Pascal Lupien (Corresponding Author)

Assistant Professor, Political Science

Brock University

1812 Sir Isaac Brock Way

St. Catharines, ON L2S 3A1 (Canadá)

E-mail: plupien@brocku.ca

Miguel Posso Yépez, Investigador

Docente de la Facultad de Educación

Universidad Técnica del Norte (Ecuador)

Av. 17 de Julio 5-21

Ibarra, Ecuador

Telephone: $(+593) 994832470$

E-mail: maposso@utn.edu.ec

Andrea Basantes, Investigadora

Docente de la Facultad de Educación

Universidad Técnica del Norte (Ecuador)

Av. 17 de Julio 5-21

Ibarra, Ecuador

Telephone: (+593) 985683930

E-mail: avbasantes@utn.edu.ec

Gabriel Chiriboga, Investigador

Gestor Comunitario

Secretaria Técnica Plan Toda una Vida

Ibarra, Ecuador

Telephone: (+593) 993969611

E-mail: wchiriboga@todaunavida.gob.ec 
Resumen: Dado el hecho de que muchas communicades indígenas producen diversos tipos de productos, el uso de las TIC para promover y vender sus bienes podría proporcionarlas ingresos importantes y aumentar así su independencia económica. Si bien un número creciente de investigadores está estudiando el uso de las TIC por movimientos sociales, aún sabemos poco sobre el impacto de las TIC en los pueblos indígenas de las Américas. Nos planteamos entonces las siguientes preguntas: ¿Cuál es el impacto de las TIC en las actividades comerciales de las organizaciones indígenas? y ¿Cómo las TIC fortalecen o dificultan su capacidad para lograr la autonomía económica? Este artículo aborda estas cuestiones al examinar el uso de las TIC por parte de las organizaciones de la sociedad civil en Ecuador. Observamos que, si bien hay ventajas y desventajas, las comunidades indígenas aún no logran superar los desafíos que enfrentan con respecto a las TIC.

\begin{abstract}
Given the fact that many Indigenous communities produce various types of products, the use of ICTs to promote and sell their goods could provide them with important income and thus increase their economic independence. Although a growing number of researchers are studying the use of ICTs in social movements, we still know little about the impact of ICTs on the Indigenous peoples of the Americas. This article therefore addresses the following questions: What is the impact of ICTs on the commercial activities of indigenous organizations? and In what ways do ICTs strengthen or hinder their ability to achieve economic independence? We respond to these questions through examining the use of ICTs by civil society organizations in Ecuador. We note that while there are advantages and disadvantages, Indigenous communities are unable to overcome the challenges they face with respect to using ICTs for commercial purposes.
\end{abstract}

Key Words: Indigenous communities; economic development; ICTs; Ecuador 
Las tecnologías de información y comunicación (TIC) se han vuelto cada vez más accesibles en América Latina. Si bien un número creciente de investigadores está estudiando el uso de las TIC por movimientos sociales, los estudios suelen centrarse en casos occidentales o en movimientos urbanos en el Sur global. Aún sabemos poco sobre el impacto de las TIC en los grupos poblacionales más marginados, los pueblos indígenas de las Américas. Es más, los estudios sobre los movimientos indígenas se enfocan en casos de movilización a gran escala, pero las organizaciones de la sociedad civil indígena tienen una gama de intereses y actividades mucho más diversa, incluso el desarrollo social y el emprendimiento. En este contexto se vuelve necesario visibilizar investigaciones relacionadas a la incidencia de las TIC en la comercialización de productos en América Latina y en especial en las comunidades rurales indígenas.

Hoy en día, las TIC son herramientas claves para aumentar la capacidad productiva y la competitividad, y para la comercialización de productos (UNCTAD 2003). Es sabido que el e-commerce es un medio que permite alcanzar un público más amplio. Dado el hecho de que muchas organizaciones indígenas producen diversos tipos de productos (agrícolas, artesanales y de confección), el uso de las TIC para promover y vender sus bienes podría proporcionarlas ingresos importantes y aumentar así su independencia económica, pero nos falta evidencia empírica con respecto al tema. Ante la situación descrita, nos planteamos entonces las siguientes preguntas de investigación: ¿Cuál es el impacto de las TIC en las actividades comerciales de las organizaciones indígenas? y ¿Cómo las TIC fortalecen o dificultan su capacidad para generar ingresos?

Este artículo aborda estas cuestiones al examinar el uso de las TIC por parte de las organizaciones de la sociedad civil en Ecuador, país que ha producido un gran número de organizaciones indígenas. El objetivo del artículo es caracterizar la incidencia de las TIC en la comercialización de los productos de las comunidades rurales indígenas en el Ecuador. Observamos que, si bien hay ventajas y desventajas, las comunidades indígenas aún no logran superar los desafíos que enfrentan con respecto a las TIC; la brecha digital puede reproducir e incluso exacerbar las desigualdades socioeconómicas que ya existen entre las comunidades indígenas y no indígenas a nivel de actividades comerciales. 


\section{Las TIC y Los Grupos Sociales Marginados}

Existe una gran cantidad de literatura académica sobre el tema de los movimientos sociales indígenas en las Américas. Esta línea de investigación arroja luz sobre el surgimiento de los movimientos y sobre el uso de tácticas "tradicionales", como las marchas, barricadas y manifestaciones (Van Cott 1994, Brysk 2000, Yashar 2005, Postero 2007, Lucero 2008, Lupien 2011, Rice 2012). En Ecuador, el ciclo de protesta indígena alcanzó su pico a mediados de la década de 2000 y desde entonces parece estar en fase de desaceleración (Mijeski y Beck 2011). Este período de desaceleración coincide con la aparición de las redes sociales y un aumento espectacular en el uso de las TIC en los países occidentales. Sin embargo, hay pocos estudios sobre el uso de las TIC por movimientos indígenas en la última década.

En su influyente trabajo, el pensador español Castells $(2009,2009 \mathrm{~b})$ sostiene que el poder ahora se centra en redes organizadas en torno a los nuevos medios de comunicación. Según varios autores, asistimos a un cambio en la naturaleza misma de la acción colectiva, ya que las TIC generan campañas no dirigidas y permiten la movilización permanente sin organizaciones jerárquicas (Bennett y Segerberg, 2012, Anduiza, Cristancho y Sabudcedo 2014). Estos movimientos difusos y desestructurados han logrado reunir coaliciones potencialmente poderosas (Bennett 2003, Biekart y Fowler 2013; Langman 2005). El "individualismo" inherente en estos movimientos está reemplazando las narrativas sociales colectivas y las organizaciones jerárquicas (Bennett 2003).

Algunos autores creen que las TIC pueden ser una herramienta empoderadora para la construcción de una ciudadanía global. Argumentan que las tecnologías de comunicación reducen el costo de participación y cambian el equilibrio de poder hacia los grupos con recursos limitados (Bennett 2003, Bimber, Stohl y Flanagin 2005). Las TIC (y las redes sociales en particular) han conducido a un aumento del activismo contra el orden neoliberal y han brindado oportunidades para que los activistas puedan desafiar las estructuras de poder existentes y rodear los medios tradicionales, que a menudo distorsionan los mensajes de los movimientos sociales (Carty 2002, Pickerill 2003, Carty y Onyett 2006, Stein 2009, Biekart y Fowler 2013). Las TIC también pueden facilitar la movilización de actores que nunca han participado en la vida política al reducir las desigualdades participativas (Anduiza, Cristancho 
y Sabudcedo 2014). Esta perspectiva asume que estas personas tienen acceso a tecnologías e infraestructura adecuada.

Otros investigadores tienen un punto de vista escéptico del impacto de las TIC. Norris (2001) duda que las tecnologías logren ahondar la brecha entre quienes tienen los recursos para utilizar las TIC de manera efectiva y quienes no tienen las mismas capacidades. Señalando que se requiere acceso básico antes de que los actores marginados puedan aprovechar los beneficios potenciales de las tecnologías digitales, Norris sugiere que pueden servir para reforzar la brecha entre ricos y pobres. Norris e Inglehart (2013) encuentran disparidades persistentes, desde el acceso al hardware hasta la velocidad de las conexiones a Internet.

La mayoría de los estudios sobre el uso de las TIC por parte de los movimientos sociales se centran en las democracias occidentales (Breuer y Groshek 2014). Los pueblos indígenas del mundo están en gran medida ausentes de la discusión. Una excepción notable es el uso de Internet por parte del Ejército Zapatista de Liberación Nacional (EZLN) a principios de los años noventa. Los simpatizantes del movimiento mexicano crearon una red transnacional que les permitió a los activistas usar Internet para presionar al gobierno mexicano a través de la creación de una gran cantidad de sitios web que se enfocan en varios temas sociales y económicos promovidos por los zapatistas (Cleaver 1998, Garrido y Halavais 2003). Sin embargo, es importante señalar que las redes zapatistas se basaron en gran medida en los sitios web creados por ONG extranjeras; todavía nos falta una comprensión de cómo los movimientos indígenas pueden crear y usar las TIC de manera independiente, sin el apoyo de estos actores transnacionales. Además, los estudios que existen sobre el uso de las TIC por movimientos sociales de grupos marginados suelen enfocarse en la movilización.

La movilización es un elemento clave del repertorio estratégico de los movimientos sociales, pero no es el único. Las organizaciones también pueden acceder a la tecnología para mejorar su situación economía mediante, por ejemplo, la comercialización más efectiva de sus productos tanto en el país como en el extranjero. El emprendimiento indígena de la mano del emprendimiento social surge como iniciativa de uno o varios individuos, que pretenden encontrar soluciones a problemas sociales. El emprendedor social identifica oportunidades que se presentan a sí mismas como problemas que requieren soluciones y se esfuerzan por crear emprendimientos para resolverlos (Sullivan 2007). Un estudio realizado en Ecuador demuestra que el uso de las TIC por organizaciones indígenas depende mucho de fondos 
estatales, lo que restringe su capacidad de aprovechar al máximo las TIC para participar de manera más eficaz en la vida política y social (Lupien y Chiriboga 2017). El uso de la TIC para generar ingresos sería entonces una manera de fomentar su autonomía frente al estado. Por este motivo, necesitamos recopilar más datos empíricos sobre las condiciones que mejoran o disminuyen la capacidad de los grupos indígenas para hacer esto.

\section{Los Pueblos Indígenas, La Pobreza y Las TIC en el Ecuador}

En el Ecuador, la población predominante es mestiza e hispanohablante (82,2\% del total de habitantes). En el mismo territorio cohabitan 14 nacionalidades y 18 pueblos, algunos de ellos con lenguas propias. Particularmente, en las zonas de integración fronteriza habitan 11 nacionalidades y pueblos indígenas, de los cuales 7 son transfronterizos: Shuar, Achuar, Shiwiar, Andwa, Siekopa'i, Sápara y Kichwas, ubicadas hacia la Amazonía. Según el Instituto Nacional de Estadísticas y Censos (INEC, 2010) la población indígena alcanza un 7\% del total de ecuatorianos, con 1'018.176 habitantes. La mayoría de población indígena se centra en la zona centro del país en las provincias de Chimborazo, Pichincha, Imbabura y Cotopaxi, sin embargo, la mayor concentración de población indígena a nivel cantonal se observa en la región de la Amazonía (Gerimundia 2015).

La situación de pobreza y marginación de los pueblos indígenas ecuatorianos, como en toda América latina, siempre fue una constante desde la conquista española. La pobreza se extiende en grupos histórica y estructuralmente marginados, tales como mujeres, poblaciones indígenas, montubias y afroecuatorianas y personas en situación de movilidad humana o refugio $(2017,54)$. Los estudios e investigaciones sobre poblaciones indígenas muestran un desequilibrio importante pues abundan en temas sobre pobreza e inequidad y son débiles en lo relacionado con el emprendimiento económico (Chávez 2016, 10). Según cifras del Banco mundial, en el mundo, hay alrededor de 300 millones de personas que pertenecen a pueblos nativos, que equivale aproximadamente el 4,5\% de la población mundial, representan cerca del $10 \%$ de los pobres (Banco mundial 2018). Esto también nos lleva a tener come efecto de los años de marginación y limitaciones para dichos pueblos por parte de organismos estatales e interventores en el desarrollo de dichos pueblos. 
Desde el punto de vista normativo o legal existen avances significativos que han permitido de alguna manera la reivindicación de estos pueblos, pero claro, no siempre la normativa sea podido aplicar por una serie de factores socioculturales, económicos y políticos de los estados. La Declaración de los derechos humanos, en sus artículos 9 y 33 se afirma que los pueblos y los individuos indígenas tienen derecho a pertenecer a una comunidad o nación indígena, de conformidad con las tradiciones y costumbres de la comunidad o nación de que se trate, y a determinar su propia identidad (Naciones Unidas 2013, 2)

La nueva Constitución de la República del Ecuador (2008) en varios de sus artículos protege y motiva el desarrollo de los pueblos indígenas; de manera particular en lo referente al uso de las TIC textualmente manifiesta en su Art. 16.- Todas las personas, en forma individual o colectiva, tienen derecho a: 1. Una comunicación libre, intercultural, incluyente, diversa y participativa, en todos los ámbitos de la interacción social, por cualquier medio y forma, en su propia lengua y con sus propios símbolos. 2. El acceso universal a las tecnologías de información y comunicación.

Como evidencia de un estado con características socioculturales similares al ecuatoriano, en el caso colombiano con relación a las políticas de inclusión de los pueblos indígenas en materia de TICS en el Plan de Acción 2010, se estableció dentro del proceso de gestión de proyectos sociales, la acción estratégica de "Implementación de estrategias de alfabetización y disminución de la brecha digital” (Ministerio de Tecnologías de la Información y las Comunicaciones 2010, 78).

Con respecto al desarrollo productivo de las comunidades indígenas y como consecuencia de la normativa vigente y de la situación de pobreza de los grupos histórica y estructuralmente marginados, como lo son las mujeres, poblaciones indígenas, montubias y afroecuatorianas y personas en situación de movilidad humana o refugio, el Plan Nacional de Desarrollo 20172021 (CNP, 2017), en su objetivo 6, manifiesta que es necesario "desarrollar las capacidades productivas y del entorno para lograr la soberanía alimentaria y el Buen Vivir Rural” (p. 84). Es evidente que en el caso ecuatoriano en la última década se hicieron importantes intervenciones públicas para mejorar las condiciones de vida de la población rural más desfavorecida. Hay mejoras en la calidad de vida de la población, nuevas actividades generadoras de ingresos, mayor cultura empresarial, mejor infraestructura de conectividad, 
presencia de campesinos e indígenas en las esferas de decisión, y cambios en las relaciones de género, por medio de normas o respuestas organizacionales.

A pesar de lo expuesto en el párrafo anterior, es evidente que está pendiente consolidar un desarrollo económico rural que genere trabajo en condiciones dignas, con acceso justo a tierra, agua y factores de producción, que promueva el respeto y promoción de formas organizativas locales, la implementación de formas de producción agro productiva no intensivas en el uso de agroquímicos o dependientes de semillas certificadas, que permita incrementar los ingresos familiares y romper el ciclo de pobreza y desigualdad, que promueva una comprensión propia del campo y sus realidades, entendiendo su manera de experimentar y vivir la modernidad, manteniendo y valorizando sus saberes. Solo de esta forma se saldará la deuda histórica que se tiene con esta población.

En términos sociales, la pobreza multidimensional muestra una mayor severidad en las provincias de la Amazonía, región donde existen mayores dificultades para la dotación de servicios básicos y sociales por la gran dispersión de la población. Según el Plan Nacional de Desarrollo (CNP, 2017), sin embargo, la medición de la pobreza en estas zonas debe ser analizada con algunas consideraciones, principalmente culturales, que no empatan necesariamente con la forma estandarizada de definir pobreza (pág. 113).

La plurinacionalidad e interculturalidad del país son factores relevantes en la planificación territorial "La pobreza por Necesidades Básicas Insatisfechas (NBI) afecta dos veces más a los hogares indígenas y 1,39 veces más a las familias afroecuatorianas, en comparación con aquellas de origen mestizo; en cuanto a la pobreza extrema, hay una incidencia 2,92 veces mayor en los hogares indígenas y 1,52 más alta en los hogares afroecuatorianos, en contraste con las familias mestizas (Desarrollo, Secretaría Nacional de Planificación 2014, 33)

Si se toma en cuenta que una de las maneras para que los pueblos indígenas salgan de la situación de pobreza es el comercio y de manera específica el comercio de productos agrícolas, las prácticas económicas tradicionales no contribuyen a mejorar la economía de los pueblos indígenas porque normalmente los excedentes de los productos agropecuarios cultivados son insuficientes para el comercio y para salir de la pobreza. Normalmente en estos pueblos la producción agrícola comunitaria es utilizada para la subsistencia familiar (García Izaguirre y Arguello Mendieta 2012). 
Las TIC se desarrollan a partir de los avances científicos producidos en los ámbitos de la informática y las telecomunicaciones. Las TIC son el conjunto de tecnologías que permiten el acceso, producción, tratamiento y comunicación de información presentada en diferentes códigos (texto, imagen, sonido) (Belloch Ortí 2012). La manera en que las TIC permiten nuevas formas de registrar, almacenar y difundir datos, ha generado cambios en los sistemas de tal manera que estamos en la era de la información e inmersos en la llamada sociedad del conocimiento (Bernal Camargo y Murillo Paredes 2012). En este contexto el Ecuador ha iniciado e implementado una serie de estrategias tendientes al desarrollo de las TIC tal es así que se plantea "propiciar la asequibilidad de los bienes y servicios TIC por parte de la población, a través de mecanismos que promuevan la sana competencia en el mercado y faciliten el acceso a estos bienes y servicios" (Ministerio de Telecomunicaciones 2016, 31).

Lamentablemente un sector representativo de la población, en especial los pueblos indígenas, tendrán dificultades para acceder a las TIC, fundamentalmente por existir altos porcentajes de analfabetismo digital. Existen todavía muchas inequidades respecto a las TIC entre el campo y la ciudad. En Latinoamérica, aunque el acceso general a Internet ha mejorado considerablemente en la última década, incluso en Ecuador, ya que el acceso al internet alcanzó el 57.27 por ciento en 2017; sin embargo, los factores socioeconómicos siguen impactando el acceso a Internet en el país. En 2017, alrededor del 46.6 por ciento de los hogares en las áreas urbanas tenían acceso a internet en comparación con el 16.6 por ciento en las áreas rurales, donde viven la mayoría de las poblaciones indígenas (MINTEL, 2017). Entonces, si bien las TIC tienen un enorme potencial para apoyar las actividades comerciales de las comunidades indígenas y, por lo tanto, para generar ingresos, todavía existen barreras de acceso significativas.

\section{Metodología}

La población objeto de estudio son las organizaciones de la sociedad civil indígena ecuatoriana. Ecuador nos sirve como un estudio de caso para probar la teoría relacionada con el uso de las TIC por parte de grupos indígenas. Los pueblos indígenas ecuatorianos tienen una larga historia de movilización política y social y han desarrollado algunas de las organizaciones indígenas más importantes de América Latina (Van Cott, 2008). Las organizaciones indígenas ecuatorianas varían en tamaño, objetivos y afiliación política e incluso se encuentran en todo el país. También reflejan las divisiones geográficas y culturales que se encuentran en la mayoría de los países andinos (por ejemplo, divisiones entre 
comunidades indígenas de tierras altas y tierras bajas). Las organizaciones indígenas de Ecuador, por lo tanto, nos permiten producir una muestra útil para examinar el impacto de las TIC en sus actividades comerciales. Dado que existen cientos de organizaciones indígenas en el país, seleccionamos una muestra de 30 organizaciones pertenecientes a los pueblos indígenas del Ecuador. En la medida de lo posible, hemos analizado una muestra representativa de organizaciones indígenas, seleccionando casos según un método de casos diversos para capturar una serie de dimensiones importantes: tipo (organizaciones políticas, sociales, comunitarias, cooperativas), tamaño, los diferentes pueblos y naciones indígenas y distribución de la población (Costa, Sierra y Oriente).

Se seleccionó un diseño de estudio de caso cualitativo como el medio más apropiado para abordar las preguntas de investigación. Centrarse en un número limitado de casos permite una descripción detallada y comparaciones de casos; es el diseño más práctico para evaluar el impacto de las TIC dentro de las organizaciones, ya que le permite al investigador usar una serie de métodos de recopilación de datos cualitativos para entender los procesos y las experiencias de los participantes.

La investigación se realizó entre junio de 2016 y febrero de 2018. Los datos son producto de una encuesta y una serie de entrevistas semiestructuradas. El instrumento primario utilizado fue un cuestionario (entrevista semiestructurada y a profundidad) que consta de 8 variables (Comunicación, Influencia en la vida pública, Influencia en las políticas públicas, Movilización de los simpatizantes, Movilización de los recursos, Desarrollo de redes y alianzas, Promoción de identidad colectiva, y Comercialización de productos). En este artículo, nos enfocamos en la última variable: comercialización de los productos de las comunidades rurales indígenas. Preguntamos acerca de las tecnologías que usan para promover y vender sus productos. El instrumento fue validado por seis expertos en el área y por usuarios mediante la aplicación de tres entrevistas piloto; también se utilizó un diario de campo, fichas de observación y cámara fotográfica en cada una de las entrevistas.

Se realizaron veinte tres (23) entrevistas semiestructuradas con un subconjunto de encuestados. De cada una de las organizaciones se seleccionaron a los líderes, todos ellos considerados como informantes calificados para la presente investigación. El propósito de las entrevistas era lograr una comprensión más profunda de algunos problemas complejos que no 
podían evaluarse adecuadamente por los cuestionarios, permitiendo a los participantes discutir sus experiencias concretas con el uso de las TIC en sus organizaciones. La investigación desarrollada fue aprobada por el Consejo Directivo del Centro de Investigaciones Científicas y Tecnológicas de la Universidad Técnica del Norte (UTN, Ibarra, Ecuador) y por el Comité de Ética de la University of Alberta (Canadá). Para la aplicación de la entrevista desde noviembre de 2017 a julio de 2018, previa autorización de los informantes de cada organización comunitaria, se les explicó el objetivo de la entrevista y del proyecto, para inmediatamente solicitarles voluntariamente la firma del respectivo consentimiento informado. Los datos fueron recolectados, en aproximadamente una hora en cada una de las entrevistas desarrolladas en los hogares de los informantes o en las casas comunales de los lugares visitados; es necesario indicar que a pesar de tener la entrevista estructurada, en la mayoría de las ocasiones, por la dinámica del tema, la idiosincrasia y respeto hacia los informantes, se tuvo que transformar en un diálogo informal, direccionando el diálogo en función de los indicadores preestablecidos.

Una vez recopilada la información con los diferentes instrumentos, se procedió a transcribirlas textualmente, para luego analizarla, compararla, ordenarla y caracterizarla. Paralelamente se desarrolló una síntesis que permite construir nueva teoría a partir de la ya existente. La información teórica relevante sobre las temáticas analizadas fue tomada de autores vigentes a través de citas textuales y de autor.

\section{Hallazgos}

El panorama general es que el uso de las TIC por parte de organizaciones y movimientos sociales indígenas y es todavía muy limitado y su impacto es mínimo. Sin embargo, las organizaciones indígenas ven la importancia de la tecnología y casi la totalidad de las incluidas en este estudio respaldan el uso de las TIC. Los dirigentes consideran que en la actualidad es imposible estar completamente integrados a la sociedad (y al mercado) sin tecnologías; casi todos expresaron su interés en la innovación, y valorarían en gran manera a cualquier persona o proyecto que pueda traer innovación como apoyo a sus comunidades. Esto incluye el uso de las TIC para comercializar productos indígenas y reforzar las iniciativas emprendedoras en las comunidades. 


\section{Productos indígenas y sistemas de comercialización}

Un punto importante que se debe señalar es la presencia de diferentes tipos de producción dentro de las comunidades indígenas. En la mayoría de las organizaciones investigadas, se evidencia la producción de varios tipos de bienes y servicios. De hecho, de las 30 organizaciones con las que trabajamos, todas menos cuatro están involucradas, directamente o indirectamente, en la venta de algún tipo de producto o servicio. Estos se pueden dividir en productos primarios (agrícolas, pecuarios), secundarios (artesanías, tejidos, vestuarios) y terciarios (servicios turísticos y de alimentación). En muchos casos, los ingresos obtenidos de estas actividades son vitales para la capacidad de la organización de llevar a cabo sus funciones.

El problema dentro de las organizaciones y comunidades indígenas no radica entonces en la falta de producción, sino en temas de comercialización. Esto toma muchas formas diferentes, pero la mayoría de los líderes indígenas reconocen las deficiencias en los métodos de comercialización utilizados en sus comunidades y buscan soluciones, algunas de ellas relacionadas con las TIC, para competir con los productores comerciales. El sistema de comercialización más utilizado es el de forma directa, el cual también trae efectos a la situación económica de las poblaciones indígenas, muchas veces el desconocimiento les lleva cometer errores dentro de sus procesos de negociación o comercialización.

Observamos tres modelos de venta directa: las transacciones a través de un acopiador o mayorista, la oferta en mercados y ferias, y la venta de forma personal o privada. En el primer caso, el nivel de negociación se ubique en el acopiador o mayorista, lo que incide que en el proceso de mercadeo primario se registren diferencias estructurales entre la oferta representada por los productores y la demanda conformada por los comerciantes locales, quienes imponen condiciones en cuanto a cantidad, calidad, precios y formas de pago. Estas transacciones generalmente implican una dinámica de poder, en la cual el mayorista se favorece a ganar frente al productor, por tener más recursos, ventajas y experiencia en el proceso de negociación. 
Alrededor de la mitad de las organizaciones entrevistadas venden productos en mercados locales o ferias parroquiales. Si bien este método tiene la ventaja de eliminar al intermediario y permitir que los productores vendan directamente a los consumidores (y así desarrollar una base de clientes leales), los líderes indígenas lamentan que la mayoría de los productores no manejen las reglas del mercado, lo que puede desfavorecerlos con respecto a aquellos que tienen más experiencia de comerciante. Para muchas comunidades, los turistas extranjeros representan una importante base de clientes, ya que tienden a frecuentar mercados y ferias en ciertas partes del país. Algunos mercados indígenas ecuatorianos se han hecho "de rigor" en el circuito turístico, ya que los extranjeros acuden a estos lugares para comprar productos artesanales, ropa, y material artístico.

El tercer modelo de venta directa es de forma personal o privada: se trata de un individuo (generalmente un líder o dirigente) que vende productos hechos por su familia directamente a un cliente, sin pasar por la organización. Si bien esta forma de venta directa parece ser cada vez más común, no permite que la comunidad se beneficie de la experiencia adquirida a través de las transacciones.

Algunas organizaciones indígenas están abordando las deficiencias en los métodos de comercialización por la creación de corporaciones de productores. El dirigente de una federación campesina de Imbabura (provincia en el norte del país) explica: "Nos organizamos con el fin de eliminar el intermediario y por mantenernos unidos. Con esa corporación de productores, podemos comprar máquinas para desespinar la frutas, preparar el producto, cosas así”. Los dirigentes también comisionaron a una empresa para preparar un estudio de mercado. La Federación Shuar, en la región amazónica, busca ayudar a sus socios a comprender el funcionamiento del mercado y a desarrollar las habilidades que necesitan para producir y vender de manera más eficiente. El representante explica:

"Muchas familias solo hacen las ventas en los mercados, o van a la ciudad. Tienen esa iniciativa, ese emprendimiento, pero se requiere un apoyo técnico o financiero. Les ayudamos en la parte técnica, para que puedan producir, para hacer contratos, para ayudarles a fortalecer o industrializar estos productos que ya están cultivando. Buscamos canales de comercialización.” 
La Confederación Nacional de Organizaciones Campesinas, Indígenas y Negras (FENOCIN), por su parte, está desarrollando un proyecto, con la ayuda de una ONG internacional, con el fin de elaborar un programa de capacitación sobre temas como emprendimiento empresarial, procedimientos, y valor agregado del producto. Otras organizaciones, como la Federación de la Nacionalidad Shiwiar de Pastaza, aprovechan del apoyo de redes o alianzas nacionales o buscan a vincularse a organizaciones internacionales para mejorar su visión para los negocios, o para ampliar sus oportunidades de promoción. En muchas comunidades, sin embargo, el aparato de comercialización sigue siendo muy básico. Según señala Oswaldo Echeverría, uno de los lideres entrevistados

"En el caso de los bordados hay una o dos familias que se dedican a la confección y tienen sus negocios internos y venden a su círculo de clientes y más en época de fiestas. En pesillo no hay producción, lo que por lo tanto no se utilizan pasa es que el sistema económico está basado en la producción agrícola y ganadera herramientas tecnológicas."

Una de las actividades a la cual muchas organizaciones y comunidades señalan que puede ayudarles a salir de la pobreza es el turismo, el cual se puede potenciar a través de promociones y ventas a través de medios tecnológicos y de información. A pesar del considerable potencial, esta actividad presenta hoy pocas comunidades con potencialidad de desarrollo y muchas experiencias marcadas por la incertidumbre debido a que la mayoría de las actividades turísticas han sido aglomeradas hasta la actualidad por la empresa privada. También señalan problemas existentes, en relación al turismo comunitario; la dispersión de los programas entre las diversas instituciones genera un sentido de confusión en las comunidades, las cuales cuestionan la actuación de los diferentes intermediarios.

Algunas organizaciones de turismo comunitario están colaborando con comunidades indígenas para que sean escuchadas como grupo y no como personas individuales, lográndose lentamente el empoderamiento de las nacionalidades y pueblos, y una identidad frente a la defensa del territorio. Sin embargo, resulta evidente que la situación actual, de distintos enfoques o formas de hacer por parte de los principales actores implicados, está ralentizado considerablemente el alcance de sus principales objetivos (ver García Palacios , 2016, pág. 611). En la mayoría de los casos con los que trabajamos, los líderes ven margen de mejora con respecto a la comercialización de productos y servicios indígenas, y la mayoría cree que las TIC podrían ayudar, aunque a menudo no saben de dónde empezar. 
Las TIC y los sistemas de comercialización: usos, usos potenciales y desafíos

Las tecnologías más utilizadas en las comunidades indígenas ecuatorianas son: teléfonos celulares, computadoras, correo electrónico, sitios web y cuentas de redes sociales como Facebook. Los teléfonos celulares son muy comunes, y son usados tanto por las organizaciones indígenas como por la gente de las comunidades en las que sirven. Existe cierta variación entre las organizaciones; algunas afirman que todos o la mayoría de sus miembros tienen teléfonos celulares, mientras que otros afirman que algunos de sus miembros no. Sin embargo, en general, son el medio más frecuente de comunicación. Los líderes utilizan teléfonos celulares para comunicarse entre sí y con sus miembros.

Todas menos dos de las 30 organizaciones tienen computadoras en su sede central, y acceso a Internet y a correo electrónico. Estos son principalmente modelos de escritorio más antiguos; once organizaciones tienen computadoras portátiles. Las computadoras fueron adquiridas a través de propuestas de financiación, o fueron donadas por otras organizaciones. De las 30 organizaciones, ocho no tienen acceso a internet en sus oficinas. Esto se explica a veces por el costo, aunque a veces se relaciona con la falta de infraestructura adecuada en las comunidades rurales. Sin embargo, en todos menos uno de estos casos en los que Internet no estaba disponible en la oficina de la organización, la persona que entrevistamos tiene acceso desde su hogar y lo usa para enviar correos electrónicos o actualizar las redes sociales en nombre de la organización.

El correo electrónico se utiliza para enviar documentos, para la comunicación entre las distintas organizaciones (tales como FENOCIN) y para convocar reuniones. También se utiliza para comunicarse con los donantes y los organismos gubernamentales, aunque no con sus propias comunidades debido a la falta de acceso. Todas menos seis de las organizaciones tienen un sitio web. La mayoría de los sitios web tienen sólo contenido básico y son muy estáticos (no han sido actualizados desde hace años).

La mayoría de las organizaciones (26) tienen cuentas en las redes sociales (Facebook siendo el más utilizado de estos sitios), aunque algunas las usan con más frecuencia que otras. En general, las páginas de Facebook de las organizaciones indígenas tienen un número moderado de "seguidores"; entre 500 para las pequeñas organizaciones y 25,000 para las más grandes. 
Una revisión de las páginas de Facebook de las organizaciones muestra un modesto nivel de interacción. A veces las personas dejan comentarios y sugerencias, o muestran su apoyo u oposición a algo que la organización está haciendo. Pocas organizaciones tienes cuentas Twitter o en sitios para compartir vídeos (YouTube, Vimeo).

Como se expone anteriormente, las comunidades y organizaciones indígenas ecuatorianas producen diversos tipos de bienes, y los productos son comercializados de forma directa, sin recurrir a procesos de promoción. Existen casos en los cuales principalmente en el tema de servicios y artesanías si se ha realizado promociones, aunque de una forma limitada, o a su vez de manera personal, mas no a través de las organizaciones. Al referirse a temas de comercialización Apawki Castro representante de la Confederación de Nacionalidades Indígenas del Ecuador (CONAIE) para temas de comunicación señala, “como organización no, como CONAIE no, como persona se ha realizado pero esas son otras experiencias".

Entre las organizaciones que han utilizado las TIC para sus actividades comerciales, observamos cuatro tipos de usos. El primero, y el más común, consiste en anunciar la participación de la organización o comunidad en un mercado o feria a través de un sitio web o de redes sociales. Esto implica el envío de mensajes a través de Facebook o Twitter, para que las personas interesadas sepan que asistirán a una feria e información sobre qué tipo de bienes estarán disponibles. Si bien quienes usaron este medio de comunicación creen que es un medio útil para llegar a clientes potenciales, es probable que estos mensajes solo lleguen a personas que ya están vinculadas a los "amigos" en línea de las organizaciones indígenas.

El segundo uso consiste en promocionar productos específicos en las redes sociales o en un sitio web; en ese último caso, consisten en sitios web estáticos que no tienen capacidad para que los consumidores interactúen con el sitio o compren los productos. Aquí también, los entrevistados creen que las TIC son una herramienta útil para promocionar sus productos a un público más amplio, pero estos esfuerzos tienen un alcance limitado por el uso básico de las tecnologías. El tercer uso implica crear páginas en sitios como Facebook para anunciar servicios a clientes potenciales; este método es utilizado frecuentemente por quienes ofrecen experiencias de turismo comunitario. Finalmente, algunas organizaciones utilizan Internet para investigar las tendencias con respecto a los productos que venden. Una costurera de la sierra norte, socia de una cooperativa de mujeres indígena señala: "Me gusta usar Internet para conocer las tendencias de la moda, para ver qué están haciendo las demás. Puedo ver tendencias en cualquier parte del mundo. Me da ideas que puedo usar en mis diseños." No 
observamos ningún caso de usos más avanzados de las TIC para los fines, por ejemplo, de vender productos directamente a consumidores extranjeros a través de un sitio web o de recolectar dinero a través de un sitio como Pay Pal.

Una novedad interesante es el uso de Facebook para promover el turismo comunitario, y algunas de estas iniciativas han tenido bastante éxito. Pesillo (Olmedo), una pequeña comunidad al norte de Quito ha utilizado Facebook como una herramienta para llegar a los turistas que desean aprender más sobre los estilos de vida indígenas. Su página, Pesillo Nuestro, "promueve la belleza natural y cultural de la Región...para reconocimiento de nuestra identidad y riqueza." La pequeña comunidad de menos de 4,000 tiene casi 6,000 seguidores en Facebook y han visto un aumento significativo en el turismo. Cabe señalar que los pueblos Shuar, que viven en las regiones más aisladas del país (Oriente) han utilizado Facebook para promover el turismo comunitario. Por ejemplo, José Vargas Moya, un joven empresario Shuar, promueve su empresa de ecoturismo a través de su página Facebook, que ofrece videos de las actividades que los turistas pueden realizar, artículos sobre el ecoturismo y fotos de los turistas disfrutando de la selva amazónica. Aunque no hay conexiones fiables a Internet es los pueblos aislados, los empresarios que desean promover sus servicios en las redes sociales pueden dirigirse a la ciudad más cercana para acceder desde un café internet o un telecentro.

Recordando los sistemas de comercialización en las comunidades indígenas ecuatorianas, se puede contemplar varios usos potenciales. Los líderes indígenas ya están pensando en eso: la comercialización a través de las TIC fue el segundo uso potencial más discutido por los líderes indígenas, después de la comunicación. Las organizaciones ven en Internet, las redes sociales y otras tecnologías el potencial para anunciar y vender sus productos. Algunos consideraron que estas tecnologías podrían ayudarles a exportar sus productos hacia el exterior. Varios dirigentes señalaron que las TIC también pueden fortalecer la capacidad de las organizaciones para participar en tareas cotidianas, tales como el pago de facturas de todas las parroquias, la organización de la información y las tareas administrativas. El uso de software puede hacer que sea más fácil de racionalizar y gestionar todo esto, lo que deja más tiempo para otras cosas. Internet se utiliza para acceder a información sobre leyes, tendencias o noticias de actualidad que pueden impactar en la comunidad y algunas organizaciones utilizan Internet para encontrar nuevas tendencias o formas de comercializar sus productos. Teniendo en cuenta los diferentes métodos de venta directa preferidos en las comunidades indígenas, es fácil imaginar varios usos potenciales de las TIC: cursos en línea para capacitar 
a empresarios indígenas en el trato con mayoristas o en las técnicas de negociación, crear mercados y feriados "virtuales" para vender productos locales a consumidores extranjeros, agilizar el proceso de venta personal o privada.

Sin embargo, si bien las organizaciones indígenas creen que las TIC podrían ayudarlas a mejorar su producción, desarrollar sus mercados y promover sus productos, el uso de tecnologías de comunicación en estas actividades sigue siendo limitado. A pesar de un puñado de experiencias innovadoras, en su mayor parte las comunidades indígenas no se han beneficiado completamente de las TIC en sus actividades comerciales. De hecho, de las 30 organizaciones con las que trabajamos, solo cuatro utilizan las TIC con regularidad y como componente central de sus estrategias de comercialización. Algunas otras han utilizado las TIC para promocionar productos, pero de manera muy básica. Marco Toalombo, líder indígena de la sierra central señala:

"Realmente en eso debo ser muy honesto, no hemos hecho a través de redes sociales lo hemos hecho a través de ventas directas en mercados los días de la feria, en el tema de comercialización no hemos podido trabajar todavía, mentiría en decir que hemos estado utilizando la tecnología, quizá en ese sentido no hemos avanzado"

Al igual que otros líderes, explicó que existen varios desafíos en el uso de las TIC por las organizaciones indígenas y rurales en sus actividades comerciales. Después de analizar y replantear la información recolectada dentro de la presente información se ha podido focalizar dos principales problemas dentro del uso de las TICs en procesos de comercialización, el primero y quizá más evidente fue el limitado desconocimiento del manejo de tecnología. La mayoría de los líderes lamentan que sus miembros, en particular los habitantes de las comunidades rurales, no tengan acceso a estas tecnologías o no sepan manejarlas. Los representantes de las organizaciones identificaron la falta de capacitación, conocimiento y experiencia con respecto a las TIC como el principal obstáculo para el uso de la tecnología. La mayoría de los activistas indígenas y los miembros de las comunidades no tienen las habilidades necesarias para utilizar las TIC con eficacia y las organizaciones no tienen recursos para capacitar a los ciudadanos o contratar expertos técnicos. Un director explicó lo que muchos de sus compañeros nos dijeron:

"La tecnología debe aprenderse y no tenemos la capacidad de extender esto a nuestras comunidades. Así que utilizamos medios de comunicación pasados de moda, que incluyen simplemente caminar para comunicarnos en persona. Creo que esto significa 
que estamos perdiendo, nos estamos quedando atrás de otros grupos que utilizan las TIC de manera efectiva."

La falta de infraestructura adecuada en las comunidades indígenas es otro desafío. Si bien la mayoría de las organizaciones que estudiamos tienen al menos equipos básicos y acceso a Internet en sus oficinas, muchas comunidades, especialmente las áreas rurales aisladas, no pueden permitirse el lujo de llevar la tecnología a su gente. Con el apoyo del estado, algunos distritos administrativos han establecido telecentros (centros comunitarios donde las personas pueden acceder a computadoras e Internet), pero generalmente se instalan en la ciudad principal de cada distrito. Las personas de las comunidades rurales no tienen fácil acceso y con frecuencia deben viajar largas distancias a pie para acceder a ellas.

Si bien el tema de acceso y manejo de tecnologías va de la mano con la educación (y según datos del gobierno este acceso ha ido creciendo), todavía se evidencian casos de analfabetismo y analfabetismo digital. Según datos sobre la brecha de acceso a Tecnología, Ecuador es uno de los países de la región donde más se han realizado históricamente esfuerzos en torno a las TICs (Cerbino \& Richero , 2007); cabe señalar que en el sector rural el 29.9\% de la población utiliza computadora. En el área rural el incremento es de 8,4 puntos más que en el 2010 (Tecnologías de la Información y Comunicaciones 2013, pág. 10). La población indígena sigue presentando las mayores tasas de crecimiento; para el 2010 con $29.4 \%$ y para el 2011 con $27.3 \%$ (Instituto Nacional de Estadisticas y Censos, 2012), esto quizá es un dato alentador aunque dentro de la investigación realizada se evidencia que se presenta un problema generacional en temas de acceso a tecnologías. Este problema se genera debido al reciente aparecimiento de las TICs, las antiguas generaciones se encuentran en un desconocimiento total, o en algunos casos parciales, esto debido a la limitación en el acceso a las TIC así como también en algunos casos los rezagos de analfabetismo. Sin embargo, al 2013 solo el 9.1\% de los hogares del área rural tienen acceso a internet en casa. Es más, incluso cuando hay acceso a computadoras e internet (en las oficinas de las orgnizaciones más grandes, por ejemplo), observamos un desconocimiento de los alcances de las TICs, lo que lleva consigo una limitación según las actividades a los que este enfocada la organización, estos pueden ser canales de comercialización, comunicación, actualización de información, etc. 


\section{Reflexiones}

Los hallazgos expuestos en este artículo pintan un cuadro de contrastes. Por un lado, las TIC proporcionan información y herramientas a los actores con escasos recursos para que puedan alcanzar sus objetivos comerciales de manera más eficiente. Por otro lado, las organizaciones indígenas carecen de acceso, infraestructura y capacitación, lo que proporciona una ventaja a los que ya saben majear estas tecnologías. En este momento, parece que las desventajas superan los beneficios. Cuando se trata de comercio, la brecha digital puede reproducir e incluso exacerbar las desigualdades socioeconómicas que ya existen entre las comunidades indígenas y no indígenas.

Las primeras reflexiones puestas a consideración están en el marco de la pregunta investigativa ¿Cuál es el impacto de las TIC en las actividades comerciales de las organizaciones indígenas? A medida que los consumidores hacen más compras en línea, tanto en los países occidentales como en las ciudades latinoamericanas, el comercio electrónico está reconfigurando fundamentalmente el mercado minorista. Es obvio que hoy en día Internet es una herramienta ineludible para promover y vender servicios y productos, pues es un medio que nos permite alcanzar un público más amplio. La globalización y la tecnología permiten a las empresas, grandes y pequeñas, vender productos y servicios a través de las fronteras y comprometerse con clientes de todo el planeta. Estas tendencias plantean tanto desafíos como oportunidades para las comunidades indígenas y rurales. Oportunidades porque las da acceso a un mercado global, desde Quito hasta Berlín y Beijing. Desafíos porque las comunidades indígenas carecen de habilidades tecnológicas e infraestructura para competir con aquellos que pueden aprovechar estas herramientas plenamente. Cabe preguntar cómo las organizaciones indígenas, particularmente las que representan comunidades rurales, pueden transformar estos desafíos en oportunidades.

Las organizaciones indígenas han demostrado su capacidad para innovar. En el siglo XX, los pueblos indígenas andinos utilizaron el video y la radio para compartir mensajes, movilizar a los partidarios y promover la cultura y los idiomas indígenas dentro y fuera de las comunidades. Los observadores han caracterizado el video como una herramienta de empoderamiento que permite a los productores de contenido indígena recurrir a formas de comunicación ancestrales, como la narración de historias, para insertar sus narrativas culturales en la esfera pública (Lupien 2017). 
Sin embargo, las tecnologías digitales de hoy en día son mucho más complejas. Los programas de radio y los videos fueron producidos localmente con un equipo básico. Aprovechar de las TIC del siglo XXI exige la expansión de la infraestructura a los residentes rurales, la incorporación del tipo de infraestructura requerida para el acceso regular a Internet y la capacitación para manejar las TIC, pero los gobiernos locales no cuentan con recursos suficientes y es poco probable que estas inversiones provengan del sector privado, ya que las empresas no ven ganancias en la expansión del acceso a las comunidades indígenas rurales. La capacitación también implica una inversión a largo plazo.

Sirven como cursores para seguir con las reflexiones la segunda pregunta de investigación planteada: ¿Hasta qué punto las TIC fortalecen la capacidad de las organizaciones indígenas para generar ingresos? Los hallazgos expuestos en el presente artículo tienen implicaciones importantes para las comunidades y organizaciones indígenas en Ecuador y más allá. Por un lado, las organizaciones indígenas empiezan a reconocer el potencial de las TIC para comercializar sus productos y venderlos a un mercado de consumidores internacionales. Esto podría tener un impacto significativo en la capacidad de las organizaciones indígenas para participar en la vida política y perseguir sus objetivos independientemente de los actores estatales o del sector privado. Muchas, si no la mayoría de las organizaciones indígenas, dependen en gran medida de la financiación de agencias estatales, ONG o (ocasionalmente) donantes privados. Aquellas que deben su supervivencia a las subvenciones estatales evitan criticar abiertamente al gobierno y, por lo tanto, actúan con una restricción considerable cuando publican mensajes en las redes sociales. Si bien las organizaciones indígenas más grandes siempre han participado en actos de resistencia y siguen haciéndolo cuando creen que es necesario, las organizaciones más pequeñas no pueden permitirse perder los escasos fondos que reciben de las agencias públicas y, por lo tanto, practican la autocensura. La financiación de las ONG a menudo viene con condiciones; las ONG extranjeras suelen esperar que los destinatarios sigan prácticas particulares o se adhieran a las creencias religiosas o ideológicas de los donantes. Incluso cuando no dependen del dinero externo para las actividades del día a día, las organizaciones indígenas están limitadas con respecto a las estrategias y tácticas que pueden usar para perseguir sus objetivos por su falta de recursos financieros. El resultado es que la autonomía política de las organizaciones indígenas queda limitada con respecto a estos actores más poderosos. 
A pesar de esta promesa, hemos visto que el potencial de las TIC para brindar a las organizaciones indígenas una mayor autonomía económica-y, por lo tanto, política — se ve obstaculizada por falta de recursos, infraestructura y capacitación en las comunidades. Se trata entonces de un gran potencial sin explotar, y esto tiene implicaciones significativas para la sociedad civil indígena en general. Sobre la base de marcos teóricos relacionados con la movilización de recursos, las oportunidades políticas y la identidad, la literatura identifica varias fortalezas que contribuyeron a la fuerza relativa de los movimientos sociales indígenas en la década de 1990. Estas ventajas incluyen un poderoso sentido de identidad colectiva (y la capacidad relacionada de los líderes para involucrarse en políticas de identidad), organizaciones sólidas que proporcionaron liderazgo a comunidades con reclamos similares y uso estratégico de tácticas disruptivas "tradicionales" como marchas y barricadas (Yashar 2005; Lupien 2011; Rice 2012). Sin embargo, gran parte de la literatura sobre activismo en el siglo XXI sostiene que estos factores son menos importantes hoy en día y que las redes sociales y otras TIC son herramientas clave en cualquier movimiento de protesta contemporáneo. Si las redes sociales y sus marcos de acción personalizados han reemplazado las protestas organizadas y la identidad colectiva (las fortalezas de los movimientos indígenas en los años 80 y 90) y si su acceso a las TIC es limitado con respecto a otros segmentos de la sociedad y el estado, entonces parece que los movimientos indígenas se encontrarán en una situación de desventaja. Ya sea para promover actividades comerciales, autonomía política o participación ciudadana, las TIC están reforzando las desigualdades entre diferentes conjuntos de actores sociales, y pueden estar introduciendo nuevas barreras que no existían hace dos décadas. Si bien los actores marginados urbanos pueden superar estos obstáculos en cierta medida, los pobres rurales (una categoría que incluye una proporción significativa de los pueblos indígenas de América Latina) se quedan atrás. Con la finalidad de acortar estas desigualdades entre el área urbana y rural en el Ecuador, el Estado se esfuerza, es más, pretende reducir la pobreza a través de su Plan Nacional de Desarrollo y otras medidas conexas. Dado el potencial que tienen las organizaciones indígenas para generar ingresos mediante el uso de las TIC, parece que este debería ser un área de enfoque del gobierno. Lamentablemente el acceso y la alfabetización digital en las zonas rurales siguen siendo limitados; las agencias estatales deben apoyar el desarrollo de las capacidades y la infraestructura de las TIC en las comunidades indígenas como un medio de reducción de la pobreza. 
Se necesita más investigación sobre el tema del uso de las TIC para desarrollo social y el emprendimiento en las comunidades indígenas. Responder a estas preguntas contribuye a un área de teoría en desarrollo a medida que los académicos intentan reconceptualizar la acción política en la era de Internet. La teoría que está surgiendo no ha considerado adecuadamente las circunstancias únicas de los pueblos indígenas, que no solo están excluidos en términos socioeconómicos sino que tienen un conjunto distinto de demandas y reivindicaciones que giran en torno al territorio, la identidad y la autonomía. De igual importancia, esta línea de investigación ayudará a las comunidades indígenas a identificar y desarrollar soluciones para ayudarles a competir con actores económicos y políticos con mejores recursos. La inclusión de experiencias indígenas en el estudio de las TIC mejora nuestra comprensión del impacto de las nuevas formas de participación económica y cívica "en línea” de los actores marginados, ya que, en el contexto actual, los pueblos indígenas tendrán más dificultades para participar en la economía globalizada. 


\section{Bibliografía}

Anduiza, E, C. Cristancho y J. Sabucedo (2014). Mobilization through online social networks: the political protest of the indignados in Spain. Information, Communication \& Society, 17:6, 750-764.

Banco Mundial. 2018. Pueblos Indígenas. Retrieved from http://www.bancomundial.org/es/topic/indigenouspeoples/overview

Belloch Ortí, C. 2012. Las tecnologías de información y comunicación. Valencia: Universidad de Valencia.

Bennett, W.L. 2003. Communicating Global Activism: Strengths and Vulnerabilities of Networked Politics. Information, Communication \& Society, 6, 143-168.

Bennett, W.L. \& Segerberg, A. 2013. The Logic of Connective Action: Digital Media and the Personalization of Contentious Politics. Cambridge: Cambridge University Press.

------. 2011. Digital Media and the Personalization of Collective Action: Social Technology and the Organization of Protests against the Global Economic Crisis. Information, Communication \& Society, 14, 770-799.

Biekart, K. \& Fowler, A. 2013) Transforming activisms 2010+: Exploring Ways and Waves. Development and Change, 44, 527-546.

Bimber, B., Stohl, C., \& Flanagin, A. J. 2005. Reconceptualizing Collective Action in the Contemporary Media Environment. Communication Theory, 15, 365-88.

Bernal Camargo, D., y Murillo Paredes, A. 2012. El acceso de los pueblos indígenas a las tecnologías de la información y la comunicación en Colombia: ¿Inclusión o exclusión social y política? Derecho y Realidad: 193-214. 
Breuer, A y J. Groshek. 2014. Online Media and Offline Empowerment in Post-Rebellion Tunisia: An Analysis of Internet Use During Democratic Transition, Journal of Information Technology \& Politics, 11:1, 25-44,

Brysk, A. 2000. From Tribal Village to Global Village: Indian rights and international relations in Latin America. Stanford, Calif.: Stanford University Press.

Carty, V. 2002. Technology and Counter-hegemonic Movements. Social Movement Studies, $1,129-211$.

Carty, V. \& Onyett, J. 2006. Protest, Cyberactivism and New Social Movements: The Reemergence of the Peace Movement Post 9/11. Social Movement Studies, 5, 229-249.

Castells, M. 2009. The Rise of the Network Society, The Information Age: Economy, Society and Culture. Cambridge, MA; Oxford, UK: Blackwell

-----. 2009b. The Power of Identity: The Information Age: Economy, Society and Culture.

Vol II.. Cambridge, MA; Oxford, UK: Blackwell.

CEPAL. 2018. América Latina logra mejoras en salud, educación y participación política de pueblos indígenas en última década. Retrieved from https://www.cepal.org/es/comunicados/america-latina-logra-mejoras-en-saludeducacion-y-participacion-politica-de-pueblos

Cerbino, M., \& Richero, A. 2007. Gobernanza, políticas públicas y aplicaciones de Internet, FLACSO: Quito .

Chávez, M. E. 2016. Kallari Emprendimiento Indígena en Ecuador. Granada: Asociación Indígena Kallari.

Cleaver, J. 1998). The Zapatista Effect: The Internet and the Rise of an Alternative Political Fabric. Journal of International Affairs, 51, 621-64. 
Consejo nacional de planificación (CNP). 2017. Plan Nacional de Desarrollo. Quito: CNP.

Ecuador, República del. 2018. Constitución de la República del Ecuador. Quito-Ecuador .

Desarrollo, S. N. (2014). Estrategia Nacional para la Igualdad y la Erradicación de la Pobreza. Quito-Ecuador.

García Izaguirre, P., \& Arguello Mendieta, S. 2012. Economía Indígena en la Comunidad de Wasakín, Municipio de Rosita. Managua: RAAN.

García Palacios, C. 2016. Turismo comunitario en Ecuador: ¿Quo Vadis? Estudios y Perspectivas en Turismo 25:4, 597-614.

Gerimundia. 2015. Población indígena en Ecuador. Retrived from: https://gerimundia.wordpress.com/2015/04/08/poblacion-indigena-en-ecuador/

Instituto Nacional de Estadisticas y Censos . 2012. Encuesta Nacional de Empleo, Desempleo y Subempleo. Quito.

Instituto Nacional de Estadisticas y Censos. 2013. Tecnologías de la Información y Comunicaciones 2013. Quito.

Instituto Nacional de Estadisticas y Censos. 2010. VI Censo de Población y V de Vivienda. Quito.

Langman, L. 2005. From Virtual Public Spheres to Global Justice: A Critical Theory of Internetworked Social Movements. Sociological Theory, 23, 42-74.

Lucero, J. 2008. Struggles of Voice: the Politics of Indigenous Representation in the Andes. Pittsburgh: University of Pittsburgh Press.

Lupien, P. 2011. The Incorporation of Indigenous Concepts of Plurinationality into the New Constitutions of Ecuador and Bolivia. Democratization, 18 (3): 774-796. 
Lupien, P. and G. Chiriboga. 2017. Use of Information and Communications Technologies (ICTs) by Indigenous Civil Society Organizations, Information, Communication and Society. DOI: 10.1080/1369118X.2017.1397182

Mijeski, K. \& Beck, S. 2011. Pachakutik and the Rise and Decline of the Ecuadorian Indigenous Movement. Athens: Ohio University Press.

Ministerio de Tecnologías de la Información y las Comunicaciones. 2010. Proyecto Plan Vive Digital, propuesta para discusión. Bogotá: MTIC.

Ministerio de Tecnologías y de la Sociedad de la Información (MINTEL). 2017. Indicadores y Estadísticas, https://observatoriotic.mintel.gob.eclestadistica/.

Ministerio de Tecnologías y de la Sociedad de la Información. 2016. Plan nacional de telecomunicaciones y de tecnologías de la información 2016-2021. Quito-Ecuador.

Naciones Unidas. 2013. Los pueblos Indígenas y el sistema de derechos humanos de las Naciones Unidas. Nueva York y Ginebra: ONU.

Prieto, M. 2011. Espacios en disputa: el turismo en Ecuador . Quito .

Rice, R. 2012. The New Politics of Protest: Indigenous Mobilization in Latin America's Neoliberal Era. Phoenix: The University of Arizona Press.

Rovayo, J. 2008. Principios básicos de mercadeo y administración. Quito: s.n.

Ruiz Ballesteros, E., y Vintimilla, M. 2009. Cultura, comunidad y turismo. Quito: Abya Yala.

Strauss, A., \& Corbin, J. 2002). Bases de la investigación cualitativa. Técnicas y procedimientos para dessarrollar la teoría fundamentada. Colombia: Universidad de Antioquía. 
Stein, L. 2009. Social Movement Web Use in Theory and Practice: a Content Analysis of US Movement Websites. New Media and Society, 11, 749-71.

Sullivan, D. 2007. Social Enterprise: The New Economic Engine? Stimulating Social Entrepreneurship: Can Support From Cities Make a Difference? Academy of Management Perspectives, 21, 1: 77-78.

Trucco, L. 2013. The Digital Divide in the Latin American Context. In Ragnedda, M. and Muschert, G. The Digital Divide: The Internet and Social Inequality in International Perspective. New York; London: Routledge.

UNCTAD. 2003. Informe sobre el Comercio Electrónico y el Desarrollo 2003.

UNESCO. 2009. La alfabetización en el Ecuador. Evolución histórica, información actualizada y mapa nacional del analfabetismo. Quito.

UNESCO. 2009. La alfabetización en el Ecuador. Evolución histórica, información actualizada y mapa nacional del analfabetismo. Quito: UNESCO.

Vallejo , A. 2013. Identificación y caracterización de los sistemas de comercialización primeria de la producción familiar campesina en la provincia de Carchi. In S. d. Ecuador, Diámicas de comercialización para la agricultura familiar campresina: desafíos y altermantivas en el escenario ecuatoriano. Quito: 15-38.

Van Cott, D.L. 2008. Radical democracy in the Andes. Cambridge, UK; New York: Cambridge University Press.

-----. 1994. Indigenous Peoples and Democracy in Latin America. New York: St. Martin's Press.

Yashar, D. 2005. Contesting Citizenship in Latin America: the Rise of Indigenous Movements and the Postliberal Challenge. Cambridge; New York: Cambridge University Press. 